\title{
DISLOCATED NUCLEAR FRAGMENTS AFTER CATARACT SURGERY
}

\author{
PETRI TOMMILA and ILKKA IMMONEN \\ Helsinki, Finland
}

\begin{abstract}
SUMMARY
Posterior nuclear dislocation is a serious complication of cataract surgery, especially when using the phacoemulsification technique. So far, there have been only a few reports concerning the indications and timing of vitrectomy with nuclear removal as well as long-term visual outcome of these eyes. We analysed 23 consecutive patients (follow-up at least 3 months) with intravitreal nuclear remnants after cataract surgery treated with vitrectomy and removal of posteriorly dislocated nuclei. All the eyes had increased intraocular pressure pre-vitrectomy, $63 \%$ had corneal oedema, $67 \%$ marked uveitis and $26 \%$ either retinal tear or detachment. Vitrectomy was performed within 1 week in $70 \%$ of eyes. In 14 eyes $(61 \%)$ the final visual acuity was 20/40 or more. The main reason for poor visual outcome was retinal detachment $(9 \%)$. These results indicate that with vitrectomy and removal of the nucleus good visual results can be achieved in a large proportion of eyes with posterior dislocation of nuclear remnants.
\end{abstract}

Posterior nuclear dislocation is a serious complication of cataract surgery, changing a routine operation into one with potentially sight-threatening events. Therefore it is important that the treatment of this complication is optimal in order to interfere as little as possible with the goals of the original surgery.

The issues to be considered in the treatment of posterior nuclear dislocation are the indications, timing, techniques and long-term results. Considering the frequency and significance of this complication, there are relatively few published reports on these aspects of posterior nuclear dislocation. ${ }^{1-7}$ In order to evaluate further the results of vitrectomy for posterior dislocation of the nuclei we report here our experiences and long-term results in the manage-

Correspondence to: P. Tommila, MD, Helsinki University Eye Hospital, Haartmanninkatu 4 C, 00290 Hki, Finland. Fax: 358-04715100. ment of this sight-threatening complication of cataract surgery.

\section{PATIENTS AND METHODS}

We reviewed the charts of 23 consecutive patients with intravitreal nuclear fragments after cataract operation, who had been treated at the vitreoretinal unit of the Helsinki University Eye Hospital between December 1991 and January 1994. The patients were then called for a follow-up examination (measurement of the visual acuity (VA), slit lamp biomicroscopy, tonometry and indirect ophthalmoscopy) to determine the long-term visual outcome and postvitrectomy complications. Information in the hospital records was reviewed to analyse the results of the initial pre-vitrectomy examination. In patients who did not want or were not able to attend the follow-up examination ( 8 eyes; 34.8\%), the most recent information obtained from the patient's private ophthalmologist was used.

The indications for vitrectomy included large intravitreal lens remnants, elevated intraocular pressure (IOP), persistent or uncontrolled uveitis and retinal detachment (RD).

In both the pre-vitrectomy and follow-up examination the cornea was considered to be oedematous if epithelial oedema and/or Descemet's membrane folds were noted. The IOP was considered abnormal if it was $26 \mathrm{mmHg}$ or more and/or antiglaucoma medication was used. Prior to vitrectomy, uveitis was considered to be present when moderate flare and cell reaction in the anterior chamber were recorded and/or steroid drops were administered more than five times a day and/or oral steroids were used. At the latest follow-up examination post-operative uveitis was considered present if notable flare and cell reaction was present in the anterior chamber or steroid drops were prescribed by the patient's ophthalmologist. The diagnosis of cystoid macular

Eye (1995) 9, 437-441 CC 1995 Royal College of Ophthalmologists 
oedema (CMO) was made based on clinical examination.

The present study plan has been reviewed and accepted by the ethics committee of Helsinki University Eye Hospital.

All computations were performed using the BMDP statistical software program in a VAX computer (BMDP Statistical Software, Berkeley, CA 1993). Chi-squared test or Fisher's exact test were used when comparing group frequencies.

\section{RESULTS}

Twenty-three eyes of 23 consecutive patients with posterior nuclear dislocation after cataract surgery constitute the material of the present study. The mean age of the patients at the cataract surgery was 75.3 years (range 55.7-88.7 years). Eight of the patients were men $(34.8 \%)$ and 15 women $(65.2 \%)$. The number of right and left eyes was $7(30.4 \%)$ and $16(69.6 \%)$, respectively. The mean follow-up period was 0.90 years $(0.25-1.69$ years). In 13 patients (56.5\%) the follow-up time was more than 1.0 year.

In 22 eyes $(95.7 \%)$ the cataract surgery was performed using the phacoemulsification technique and in 1 eye $(4.3 \%)$ planned extracapsular cataract extraction was done. The nucleus or its fragments were dislocated into the vitreous during the phacoemulsification as shown in Table I. In 14 eyes $(60.9 \%)$ more than half the nucleus was dislocated posteriorly and in 1 eye $(4.3 \%)$ less than one quadrant of the nucleus was dislocated. Twelve eyes $(85.7 \%)$ with large nuclear fragments were operated within 1 week compared with 4 eyes $(44.4 \%)$ with smaller nuclear fragments $(p=$ 0.0517) (Table II). A significant amount of residual cortical material was noted in 16 eyes $(69.6 \%)$.

All the patients underwent a standard three-port pars plana vitrectomy. In 17 eyes $(73.9 \%)$ the nuclear fragments were removed from the vitreous space either with a fragmatome or by crushing the nuclear material between vitreous cutter and fibre-optic probe. A small bubble of perfluorodecaline was used to protect the papillomacular area in 8 of these eyes. In the remaining 6 eyes $(26.1 \%)$ the nuclear fragments were lifted from the vitreous space to the anterior chamber using perfluorodecaline, and extracted via a limbal route. The median time from

Table I. The phase of surgery when the nucleus was dislocated into the vitreous

\begin{tabular}{lcc}
\hline Phase of surgery & No. of eyes & $(\%)$ \\
\hline Hydrodissection & 2 & $(9.5)$ \\
Grooving & 7 & $(33.3)$ \\
Cracking & 7 & $(33.3)$ \\
Emulsification & 5 & $(23.8)$ \\
Total & 21 & $(100.0)$ \\
\hline
\end{tabular}

No data were available for 1 eye and 1 patient with planned extracapsular cataract extraction was excluded.
Table II. The relation of the size of the nuclear fragment and the timing of vitrectomy

\begin{tabular}{lcr}
\hline \multirow{2}{*}{$\begin{array}{l}\text { Timing of } \\
\text { vitrectomy }\end{array}$} & \multicolumn{2}{c}{ Size of the nucleus } \\
\cline { 2 - 3 } & $<50 \%$ & $>50 \%$ \\
\hline$\leqslant 1$ week & $4(44.4)$ & $12(85.7)$ \\
$>1$ week & $5(55.6)$ & $2(14.3)$ \\
Total & $9(100.0)$ & $14(100.0)$ \\
\hline
\end{tabular}

Values are the number of eyes (\%).

cataract surgery to vitrectomy was 1 week (0-53 days) (Table III).

An intraocular lens (IOL) was implanted in 5 eyes $(21.7 \%)$ during cataract surgery and in 15 eyes $(65.2 \%)$ at the end of the vitrectomy and nucleus removal. Ciliary sulcus implantation supported by capsular remnants was possible in 18 eyes $(78.3 \%)$ whereas 2 eyes were implanted with an anterior chamber IOL. In 3 eyes $(13.0 \%)$ the IOL was implanted in another operation after the vitrectomy.

The pre-vitrectomy VA ranged from light perception to $20 / 50$. Five eyes $(21.7 \%)$ had a VA of $20 / 70$ or more whereas in 10 eyes $(43.5 \%)$ the VA was less than 20/200 (Table IV).

The frequencies of pre-vitrectomy complications are shown in Table V. All the eyes had abnormal IOP before vitrectomy, two-thirds had marked uveitis and $62.5 \%$ corneal oedema.

In 6 eyes $(26.1 \%)$ a peripheral tear and/or retinal detachment was diagnosed during the vitrectomy. Four of these eyes were treated with either indirect laser or cryotherapy and in two eyes additionally an encircling procedure with fluid-gas exchange was performed.

At the latest follow-up examination 3 patients (13.0\%) had a VA of less than 20/200 (Table IV). Two of these patients had post-vitrectomy retinal detachment which remained detached due to severe proliferative vitreoretinopathy (PVR). The third eye lost vision 15 months after cataract surgery as a result of uncontrolled IOP. This patient had pseudoexfoliative glaucoma with severe disc damage diagnosed 6 years earlier. However, 14 eyes $(60.9 \%)$ had a VA of $20 / 40$ or more and in $69.6 \%$ of the eyes the final VA was better than that before cataract surgery (Fig. 1).

The main reasons for a VA of less than 20/70 are presented in Table VI. The two patients in whom the main reason for visual loss could not be defined had a

Table III. The cumulative time interval between cataract extraction and vitrectomy

\begin{tabular}{lcc}
\hline Time interval & No. of eyes & $(\%)$ \\
\hline At the same operation & 7 & $(30.4)$ \\
Within 1 week & 16 & $(69.6)$ \\
Within 2 weeks & 20 & $(87.0)$ \\
Within 1 month & 22 & $(95.7)$ \\
Within 2 months & 23 & $(100.0)$ \\
\hline
\end{tabular}


Table IV. The visual acuity before vitrectomy and at the latest follow-up examination

\begin{tabular}{|c|c|c|}
\hline VA & $\begin{array}{l}\text { VA before } \\
\text { vitrectomy }\end{array}$ & $\begin{array}{l}\text { VA at the latest } \\
\text { follow-up examination }\end{array}$ \\
\hline $\begin{array}{l}\text { NPL } \\
\text { PL } \\
\text { HM } \\
>\mathrm{HM} \text { to }<20 / 200 \\
20 / 200 \text { to }<20 / 70 \\
20 / 70 \text { to }<20 / 40 \\
\geqslant 20 / 40\end{array}$ & $\begin{array}{lr}0 & (0.0) \\
1 & (4.3) \\
5 & (21.7) \\
4 & (17.4) \\
8 & (34.8) \\
5 & (21.7) \\
0 & (0.0)\end{array}$ & $\begin{array}{rr}1 & (4.3) \\
1 & (4.3) \\
1 & (4.3) \\
0 & (0.0) \\
3 & (13.0) \\
3 & (13.0) \\
14 & (60.9)\end{array}$ \\
\hline Total & $23(100.0)$ & $23(100.0)$ \\
\hline
\end{tabular}

Values are the number of eyes (\%).

VA, visual acuity; NPL, no perception of light; PL, perception of light; HM, hand movements.

VA of 20/100 in the latest examination. One of these patients had a peripheral retinal detachment after vitrectomy but the retina was attached with reoperation.

The relation of the timing of vitrectomy to the visual outcome is shown in Table VII. If the vitrectomy was done within 1 week of the cataract extraction $68.8 \%$ of eyes had a VA of $20 / 40$ or more, whereas the corresponding figure was only $42.9 \%$ if the time from cataract surgery was more than 1 week $(p=0.239)$. Of eyes undergoing vitrectomy immediately after cataract extraction $57.1 \%$ achieved a VA of $20 / 40$ or more, compared with $77.8 \%$ of eyes which were vitrectomised 1-7 days after cataract surgery $(p=0.365)$.

The main complications at the latest follow-up examination are presented in Table VIII. One eye $(4.3 \%)$ had mild corneal oedema. In spite of slightly blurred vision in the mornings due to mild stromal thickening the VA was $20 / 20$ in the latest examination. This eye had had a secondary anterior chamber IOL implanted 6 months after vitrectomy.

Increased IOP was diagnosed in 1 eye $(4.3 \%)$. This eye had chronic glaucoma already before cataract extraction. The IOP was controlled for 3 months after vitrectomy but thereafter increased dramatically in spite of glaucoma procedures, resulting in no light perception 15 months later. Significant postoperative uveitis was found in 3 eyes $(13.0 \%)$. In all these eyes steroid drops had been used for more than 3 months. In only 1 eye was CMO detected, 11 days

Table V. Pre-vitrectomy complications in eyes in which the vitrectomy was not performed immediately after the cataract surgery

\begin{tabular}{lcr}
\hline Pre-vitrectomy complication & No. of eyes & $(\%)$ \\
\hline Corneal oedema $^{\mathrm{a}}$ & 10 & $(62.5)$ \\
Increased intraocular pressure $^{\mathrm{a}}$ & 16 & $(100.0)$ \\
Marked uveitis $^{\mathrm{a}, \mathrm{b}}$ & 10 & $(66.6)$ \\
Vitreous haemorrhage $_{\text {Retinal tear }}$ & 1 & $(4.3)$ \\
Retinal detachment & 5 & $(21.7)$ \\
\hline
\end{tabular}

${ }^{\mathrm{a}}$ This complication could not be determined in 7 eyes operated immediately after complicated cataract extraction.

${ }^{\mathrm{b}}$ Data missing for one eye.

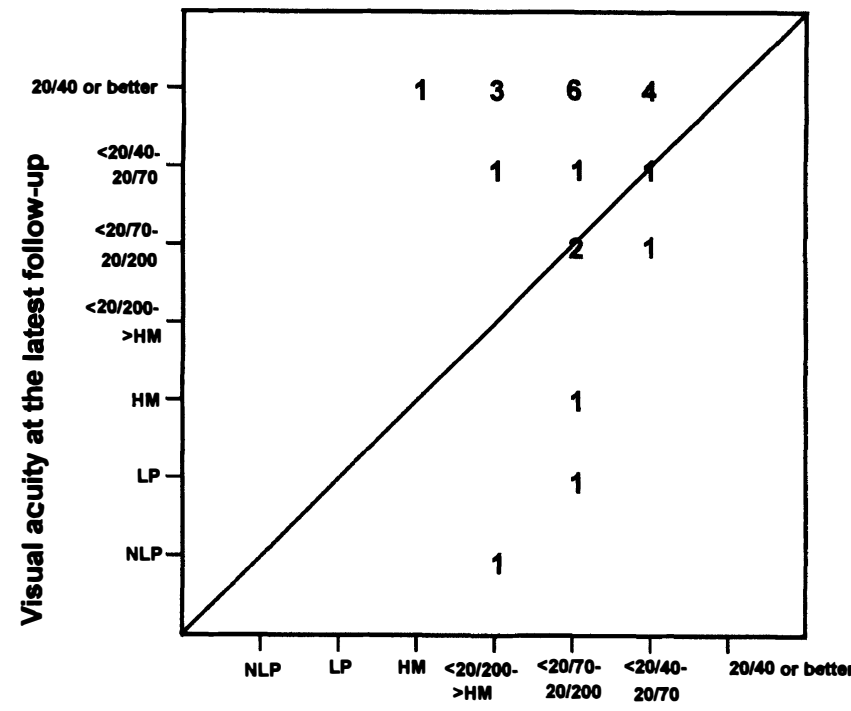

Visual acuity before cataract surgery

Fig. 1. Distribution of eyes according to visual acuity before cataract surgery and at the latest follow-up examination.

after the vitrectomy; this cleared in about 2 months. In 4 eyes it was not possible to evaluate the CMO status.

In 3 eyes $(13.0 \%)$ retinal detachment was diagnosed after vitrectomy. In 2 of these eyes a retinal tear was found and treated with cryotherapy or indirect laser during the primary vitrectomy and nucleus removal. In spite of treatment, a retinal detachment occurred 1 and 2 months after the vitrectomy, respectively. Both retinas were initially attached with fluid-gas exchange and scleral buckling, but subsequently redetached due to severe PVR. Both patients refused further operations. In the third patient retinal detachment was diagnosed 2 months after the vitrectomy. Following revitrectomy and scleral buckling the retina was reattached.

Other complications were found in 5 eyes $(21.7 \%)$ (Table VIII). In 2 eyes macular preretinal fibrosis developed in 3 weeks and 3 months after vitrectomy, respectively. In 1 eye occlusion of the inferior nasal vein of the retina was detected after retinal endophotocoagulation for a large posterior retinal tear during vitrectomy. In another eye subluxation of the IOL occurred 6 months after cataract extraction following contusion of the head. The subluxated IOL was removed and an anterior chamber IOL was

Table VI. The main reasons for a visual acuity of less than 20/70 at the latest follow-up examination

\begin{tabular}{lcc}
\hline Reason for decreased visual acuity & No. of eyes & $(\%)$ \\
\hline Age-related macular degeneration & 1 & $(4.3)$ \\
Retinal detachment & 2 & $(8.7)$ \\
Increased intraocular pressure & 1 & $(4.3)$ \\
Not defined & 2 & $(8.7)$ \\
Total & 6 & $(36.0)$ \\
\hline
\end{tabular}


Table VII. The effect of timing of vitrectomy on the final visual outcome

\begin{tabular}{lccccc}
\hline & \multicolumn{4}{c}{ Timing of vitrectomy } \\
\cline { 2 - 5 } Visual acuity & \multicolumn{2}{c}{ Same day } & \multicolumn{1}{c}{$1-7$ days } & \multicolumn{2}{c}{$>7$ days } \\
\hline$<20 / 40$ & $3(42.9)$ & 2 & $(22.2)$ & 4 & $(57.1)$ \\
$\geqslant 20 / 40$ & $4(57.1)$ & 7 & $(77.8)$ & 3 & $(42.9)$ \\
Total & $7(100.0)$ & $9(100.0)$ & $7(100.0)$ \\
\hline
\end{tabular}

Values are the number of eyes (\%).

inserted. In the fifth eye a choroidal detachment persisted for 6 weeks after the vitrectomy but cleared thereafter.

\section{DISCUSSION}

The use of phacoemulsification has increased the frequency of posteriorly dislocated nuclei in our hospital district during the last few years. Although so far there has been some debate about the proper management of this complication ${ }^{5,6}$ conservative treatment did not seem reasonable in any of our patients. Increased IOP was found in all eyes, marked uveitis and corneal oedema in two-thirds of the eyes and either a retinal tear or detachment in $26 \%$ of eyes after complicated cataract surgery. $2,3,5,7$ Moreover, most patients, who had expected a rapid visual recovery after cataract surgery, were very dissatisfied with poor vision post-operatively.

Ross $^{6}$ and Fastenberg et al. ${ }^{2}$ have described patients with good visual outcome after conservative treatment. Also Gilliland et al. ${ }^{5}$ had a few eyes with favourable visual prognosis without vitrectomy but on average the incidence of long-term complications was high and visual results not comparable with eyes that underwent vitrectomy. Blodi et $a .^{3}$ shared the same opinion. Thus, conservative treatment seems justified only in eyes with mild symptoms. ${ }^{7}$ Also, the patient must tolerate post-operative medication and have an opportunity for frequent post-operative visits.

The VA in the previtrectomy examination was less than $20 / 200$ in $43 \%$ of the eyes, which corresponds to the finding of Lambrou and Stewart ${ }^{4}(50 \%)$. Marked uveitis and elevated IOP with corneal decompensa-

Table VIII. The main complications at the latest follow-up examination

\begin{tabular}{|c|c|c|}
\hline Complication & No. of eyes & $(\%)$ \\
\hline Corneal oedema & 1 & (4.3) \\
\hline Raised intraocular pressure & 1 & (4.3) \\
\hline Chronic uveitis & 3 & (13.0) \\
\hline Cystoid macular oedema ${ }^{a}$ & 1 & $(5.3)$ \\
\hline Retinal detachment & 3 & (13.0) \\
\hline Preretinal fibrosis of the macula & 2 & (10.5) \\
\hline Branch retinal vein occlusion & 1 & $(4.3)$ \\
\hline Choroidal detachment & 1 & (4.3) \\
\hline Intraocular lens subluxation & 1 & (4.3) \\
\hline
\end{tabular}

${ }^{\mathrm{a}}$ Data missing for four eyes. tion and vitreous opacification induce this prominent visual decline. ${ }^{1,5}$

In $60 \%$ of eyes, more than half the nucleus was dislocated posteriorly in our series. This can partly explain the high frequency of IOP elevation and uveitis in our patients. Residual cortical material was present in $70 \%$ of patients, which is comparable to the figure in the series of Fastenberg et al. ${ }^{2}(71 \%)$. It appears that to avoid trauma to the peripheral retina it is better to manipulate the vitreous as little as possible at the primary cataract surgery, ${ }^{4}$ according to our experience residual cortical material had no deleterious effect and was easily cleaned during vitrectomy.

In 17 eyes $(74 \%)$ nuclear fragments were removed from the vitreous space with a fragmatome or by crushing the nucleus between vitreous cutter and the fibre-optic probe. This seems to be a feasible technique in eyes with small nuclear fragments but seemed to function well also in eyes with soft larger fragments. If most of the nucleus was dislocated or if it was very hard, it was removed with the aid of heavy fluorocarbon liquid (perfluorodecaline) via a limbal incision. Naturally; such cases require that an IOL was not implanted during the cataract surgery.

The final visual results were quite satisfactory: $61 \%$ of our patients had a VA of $20 / 40$ or more at the latest follow-up examination (Table IV), which corresponds to the figures presented in other series $^{5-7}$ (48-68\%). Moreover, most eyes experienced increased VA after vitrectomy compared with the VA before cataract surgery (Fig. 1). ${ }^{7}$ In only 3 eyes $(13 \%)$ was the final VA less than $20 / 200$, as was shown by $\operatorname{Ross}^{6}(8 \%)$ and Kim et al. ${ }^{7}(16 \%)$. Most of the post-vitrectomy complications had only limited impact on the long-term visual outcome. Retinal detachment was an exception. ${ }^{7}$ Two of 3 eyes with post-vitrectomy retinal detachments suffered redetachments as a result of PVR. As the risk of PVR after ordinary retinal detachment is about $10 \%,{ }^{8}$ it appears that in eyes with posterior nuclear dislocation and retinal detachment or tear the risk of developing PVR is higher. ${ }^{2,3,7}$ Intraocular inflammation caused by previous surgery and intravitreal nucleus material probably contribute to the development of PVR in these eyes.

Moreover, the incidence of iatrogenic retinal tears and/or detachment seems to be more frequent in eyes that underwent vitrectomy for nucleus dislocation $(26 \%)$ as opposed to vitreous haemorrhage (3$10 \%) .{ }^{9,10}$ It is not possible to tell whether these retinal tears occurred during the original cataract surgery or the nucleus removal. In any case it is important to minimise manipulation of the vitreous in the primary surgery. Also careful examination of the retinal periphery is important at the end of the vitrectomy for nucleus removal. 
In the present series the visual prognosis seemed to be better if the vitrectomy was done within 1 week of the cataract surgery rather than later. However, other series have not verified this result. ${ }^{5-7}$ However, as the number of patients was limited in the present series large differences are needed to reach statistical significance. Especially good visual results were obtained by Kim et al. ${ }^{7}$ among patients in whom the vitrectomy was performed on the same day as the cataract surgery. Although we could not confirm this, it seems advantageous to perform vitrectomy immediately after cataract extraction if possible: the patient then has only one operation instead of two procedures. However, such interventions are possible only in hospitals where vitreoretinal services are readily available.

A mild persistent corneal oedema was diagnosed in only 1 eye in our patients, suggesting that the risk of corneal decompensation after nucleus removal is not high.?

Post-operatively increased IOP was found only in 1 patient $(4.3 \%)$, and he had had pre-existing glaucoma. According to Fastenberg et al. $^{2}$ and Blodi et al. $^{3}$ the risk of chronic glaucoma on longterm follow-up is increased if vitrectomy was performed more than 3 weeks after cataract extraction. Kim et al. ${ }^{7}$ did not find such a relationship although most eyes with difficult glaucoma needing filtering surgery were among eyes with the longest interval between cataract surgery and vitrectomy. In the present study also, the only patient with postoperative uncontrolled IOP had the longest time interval between cataract surgery and vitrectomy (53 days).

The incidence of post-operative uveitis $(13 \%)$ in our patients is intermediate between that found by Blodi et al. ${ }^{3}(25 \%)$ and Kim et al. ${ }^{7}(2 \%)$. Although in all 3 eyes with persistent uveitis in our patients topical steroid treatment was continued for more than 3 months after vitrectomy, the grade of uveitis was mild.

CMO was found in 1 patient $(5.3 \%)$ after the vitrectomy. This figure in our series could have been higher if fluorescein angiography had been done. The 2 patients in whom the reason for poor final VA could not be determined might have had subclinical $\mathrm{CMO}$.

It appears that, when treated appropriately, reasonably good visual results can be obtained in eyes with posteriorly dislocated nucleus. In order to obtain optimal results after this complication of cataract surgery, it is important that manipulation of the vitreous is minimised during the original surgery, and that the nucleus is removed within 1 week by an experienced vitreoretinal surgeon using controlled three-port vitrectomy techniques.

This work was financially supported by the Finnish Eye Foundation (Silmäsäätiö).

Key words: Cataract surgery, Intraocular pressure, Nuclear dislocation, Phacoemulsification, Retinal detachment, Vitrectomy.

\section{REFERENCES}

1. Hutton WL, Snyder WB, Vaiser A. Management of surgically dislocated intravitreal lens fragments by pars plana vitrectomy. Ophthalmology 1978;85:176-89.

2. Fastenberg DM, Schwartz PL, Shakin JL, et al. Management of dislocated nuclear fragments after phacoemulsification. Am J Ophthalmol 1991;112: 535-9.

3. Blodi BA, Flynn HW, Blodi CF, et al. Retained nuclei after cataract surgery. Ophthalmology 1992;99:41-4.

4. Lambrou FH, Stewart MW. Management of dislocated lens fragments during phacoemulsification. Ophthalmology 1992;99:1260-2.

5. Gilliland GD, Hutton WL, Fuller DG. Retained intravitreal lens fragments after cataract surgery. Ophthalmology 1992;99:1263-9.

6. Ross WH. Management of dislocated lens fragments following phacoemulsification surgery. Can J Ophthalmol 1993;28:163-6.

7. Kim JE, Flynn HW Jr, Smiddy WE, et al. Retained lens fragments after phacoemulsification. Ophthalmology 1994;101:1827-32.

8. Cowley M, Conway BP, Campochiaro, PA, et al. Clinical risk factors for proliferative vitreoretinopathy. Arch Ophthalmol 1989;107:1147-51.

9. Isernhagen RD, Smiddy WE, Michels RG, et al. Vitrectomy for nondiabetic vitreous hemorrhage: not associated with vascular disease. Retina 1988;8:81-7.

10. Smiddy WE, Isernhagen RD Michels RG, et al. Vitrectomy for nondiabetic vitreous hemorrhage: retinal and choroidal vascular disorders. Retina 1988;8:88-95. 